\title{
A Potentially Detrimental Pin1 in Cerebrovascular Pathology: Beyond its Traditionally Beneficial Regulation of Amyloid- $\beta$ and Tau
}

\author{
Jing-Zhang Wang ${ }^{1, \#, *, ~ W e n-T a o ~ D u ~}{ }^{1, \#}$, Jing Bai ${ }^{1 *}$, Xiang-Yang Zhang ${ }^{1}$, Shu-Zhen Cheng ${ }^{1}$, Yu-Hua Zhang ${ }^{2 *}$ \\ and Guang Liu ${ }^{3}$ \\ ${ }^{1}$ Affiliated Hospital, College of Medicine, Hebei University of Engineering, Handan 056002, PR China \\ ${ }^{2}$ College of Life Sciences and Food Engineering, Hebei University of Engineering, Handan 056021, PR China \\ ${ }^{3}$ Department of Cardiology, Forth Hospital of Hebei Medical University, Shijiazhuang 050011, PR China
}

\#These authors contributed equally to this study and should be co-first authors

*Corresponding author: Jing-Zhang Wang, Affiliated Hospital, College of Medicine, Hebei University of Engineering, Handan 056002, PR China

Jing Bai, Affiliated Hospital, College of Medicine, Hebei University of Engineering, PR China

Yu-Hua Zhang, College of Life Sciences and Food Engineering, Hebei University of Engineering, PR China

\section{ARTICLE INFO}

Received: 幽 October 24, 2019

Published: 幽 November 04, 2019

Citation: Jing-Zhang Wang, Wen-Tao Du, Jing Bai, Xiang-Yang Zhang, Shu-Zhen Cheng, Yu Hua Zhang, Guang Liu. A Potentially Detrimental Pin1 in Cerebrovascular Pathology: Beyond its Traditionally Beneficial Regulation of Amyloid- $\beta$ and Tau. Biomed J Sci \& Tech Res 22(3)-2019. BJSTR. MS.ID.003756.

Keywords: Alzheimer's Disease; Cerebrovascular Thrombosis/Occlusion; Pathogenic Mechanism; Pin1; Endothelial Nitric Oxide Synthase

\begin{abstract}
Cerebral vascular dysfunction causes various ischemic neurological disorders necessitating novel mechanism clarification. In this work, we presented the methods to identify nine single nucleotide polymorphism (SNP) sites of the Pin1 gene in Chinese patients suffered from cerebral infarction for the first time, suggesting that these SNPs have relatively high allele frequencies and may contribute to prevention of Pin1-related neurological diseases. Based on the preliminary interpretations, the SNPs rs2233678 $(-842 G)$ and rs2287839 $(-5185 C)$ may enhance Pin1 transcription and elicit impacts on thrombosis and atherosclerosis in brain vessels. We further firstly hypothesized a novel Pin1-eNOS-NO-cerebral vasodilatation signaling pathway, which may play a DETRIMENTAL role in cerebrovascular pathologies by blocking vascular diastole/ relaxation and leading to vascular thrombosis/occlusion in the brain. In summary, genetic polymorphisms of Pin1 may represent a new genetic basis for early diagnosis and precision medicine of cerebrovascular dysfunction, and predictably Pin1 inhibitors might have therapeutic efficacy for early prevention of cerebrovascular thrombosis and relevant neural disorders.
\end{abstract}

Abbreviations: AD: Alzheimer's Disease; eNOS: Endothelial Nitric Oxide Synthase; NO: Nitric Oxide; SNP: Single Nucleotide Polymorphism; BAECs: Bovine Aortic Endothelial Cells; HAECs: Human Aortic Endothelial Cells

\section{Introduction}

Some novel neurological studies highlight the pathogenic associations of amyloid- $\beta$, tau and genetic polymorphisms with cognitive impairment, Alzheimer's disease (AD), etc. [1-4]. To our knowledge, the pathogenic mechanisms of cognitive impairment and $\mathrm{AD}$ are very complicated. Amongst relevant risk factors, cerebrovascular infarction and microvessel ischemia play very crucial roles in the development of these neurological diseases
[5-8]. Several recent studies tried to explore potential molecular mechanisms underlying cerebral vascular infarction and their relationships with neurological disorders. For example, Raghib et al. found that calcified cerebral emboli result in vascular occlusion and acute stroke in 3 patients, and a simple thrombolysis treatment was not very effective for them [9]. Diacinti et al. described 4 rare cases with cerebral venous thrombosis, which lacks typical vascular signs 
and is difficult to be diagnosed early [10]. Akpinar et al. reported 8 patients with acute tandem occlusion stroke and performed stentassisted thrombectomy to re-perfuse brain vessels [11]. Rosen et al. investigated the cerebrovascular reactivity of 26 patients with intracranial steno-occlusive disease and evaluated the long-term beneficial effects of extracranial-intracranial bypass on cerebral blood-supply [12]. Moscote-Salazar et al. further analyzed intravenous thrombolysis and recanalization for acute ischemic stroke [13].

As can be seen, the molecular mechanisms underlying cerebral thrombus and occlusion formation are very complicated, and one major explanation is reduced cerebral vasodilatation, which necessitates further identifying novel molecular basis of vasodilative reduction. In accordance with these ideas, we intend to suggest a potential role of the peptidyl-prolyl cis-trans isomerase Pin1 in these bioactive processes.

\section{Methods}

\section{Patients Description}

A few patients with cerebral infarction (and/or coronary heart disease) were randomly selected from the Affiliated Hospital of Hebei University of Engineering in 2018 and 2019. Each of them signed the informed consent and answered the questionnaire.

\section{Blood Preparation, DNA Extraction, PCR Amplification and Gene Sequencing}

All reagents for DNA sequencing were provide by the Sangon Biotech, Shanghai. $5 \mathrm{~mL}$ peripheral blood was collected, and blood cells were separated by centrifugation $(3000 \mathrm{rpm}, 10 \mathrm{~min}$, stored at $-80^{\circ} \mathrm{C}$ ). DNA was extracted by Ezup Column Blood Genomic DNA Extraction Kit, and then PCR assays were performed. DNA sequences were measured by the 3730XL DNA Analyzer. The PCR primers for nine single nucleotide polymorphism (SNP) sites of Pin1 were shown in our previous studies [14].

\section{Results and Discussions}

\section{The Preliminary Result of Pin1 Genetic Polymorphisms in Chinese Patients with Cerebral Infarction}

According to the literature and Pin1 gene information in NCBI, we validated nine interesting single nucleotide polymorphisms (SNPs) of the Pin1 gene in Chinese patients with cerebral infarction for the first time, and the DNA sequencing examples of these varied genotypes were briefly shown in Figure 1. We further predict that high-expression Pin1 alleles may attenuate NO potentially leading to a higher cerebral thrombotic risk in human beings. Thus, Pin1 SNPs were divided into three risk-levels (Figure 1, marked as +, +++, and +++++ ): First, in Pin1 gene promoter. For the SNP 1.rs2287839, the variant $-5185 \mathrm{C}$ allele has an extremely lower affinity with the Pin1 gene suppressor AP4 (brain-specific) and may up-regulate Pin1 transcription compared with -5185G [15]; For 2.rs2233678, the $-842 \mathrm{G}$ allele positively enhances Pin1 transcription and mRNA level compared with -842C [16,17]; For 3.rs2233679, -667C may contribute to a lower Pin1 level especially in -842C-genotype individuals, which probably indicates -667 T may enhance Pin1 transcription $[16,17]$.

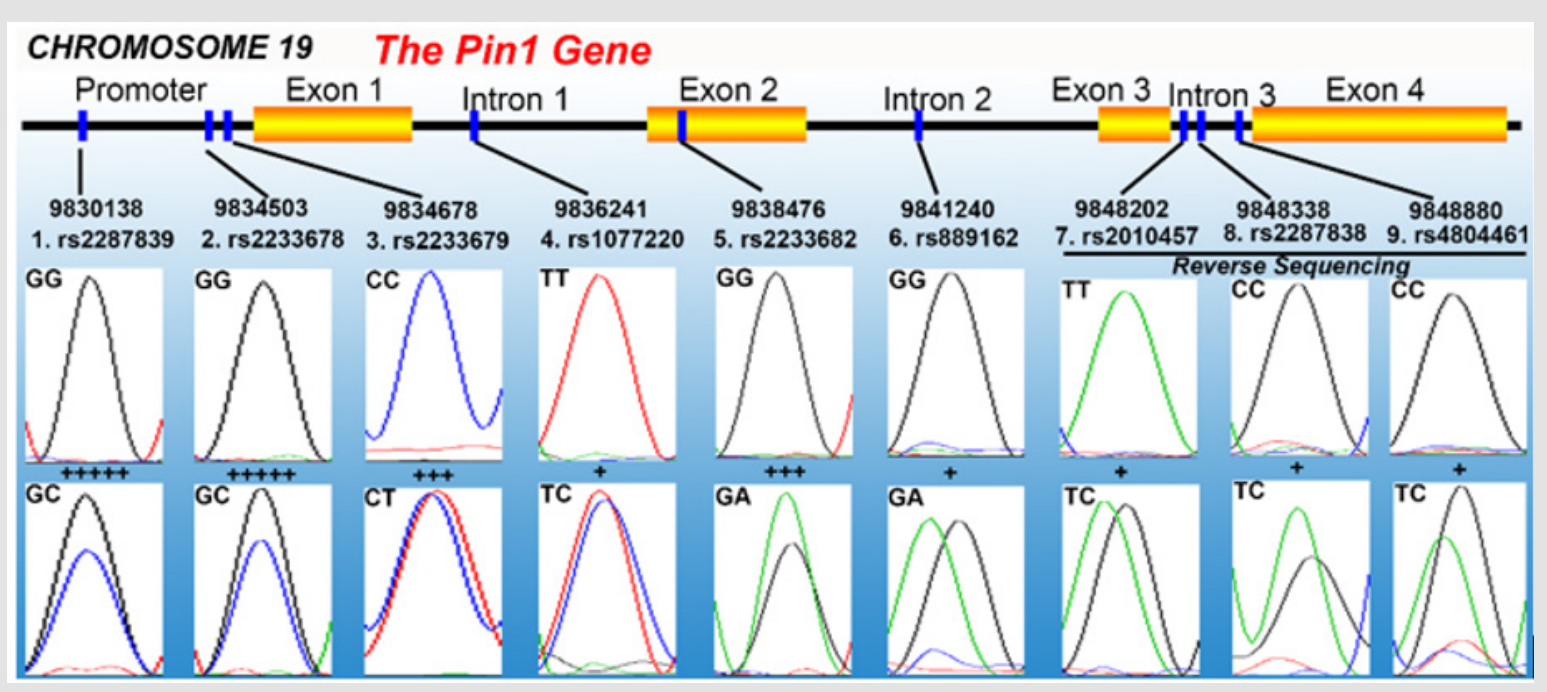

Figure 1: Examples of Pin1 genotypes analyzed in this research. The upper pictures mean the natural genotypes, and the lower pictures contain the variant genotypes. The cerebral thrombotic risks of these SNPs were predicted as three levels, marked as $(+),(+++)$ and $(+++++)$ from lowest risk to highest risk, respectively.

Second, in the exon 2 of Pin 1 gene. The SNP 5.rs2233682 results in an amino acid coding mutation from CAG to CAA, and whether this substitution disturbs Pin1 transcription and translation needs to be further verified. Third, in the introns of Pin1 gene. These SNPs may have minor influences on Pin 1 activity, but the potential impact of abundant C alleles (especially NO. 7, 8, 9 near to each 
other) on gene transcription and mRNA splicing of Pin1 also needs to be clarified.

\section{A New Pin1-Based Hypothesis for Cerebrovascular Pathology and Ischemia-Related Neurological Disorders}

Pin1 is the only known isomerase that regulates cis-trans structural transition and phosphorylation of many signaling proteins (pThr/pSer-Pro motifs) $[18,19]$. Previously, it is well known that amyloid- $\beta$ deposition and tau aggregation are two major molecular pathologies in cerebral disorders especially AD, but Pin 1 regulates processing of amyloid precursor protein and reduces amyloid- $\beta$ and tau aggregates [19-21]. In this sense, Pin1 beneficially protects brain tissues from $\mathrm{AD}$ and relevant neurological disorders. However, on the basis of a novel cerebral vasodilatation signaling pathway, we herein underline a potentially detrimental link between Pin1 and cerebrovascular thrombosis-related neuropathies. Nitric oxide (NO) has an evident vessel-relaxing/vasodilatation bioactivity and is a key target of cardiovascular drugs [18,22]. However, Ruan et al. revealed that Pin 1 inhibits the endothelial nitric oxide synthase (eNOS) in bovine aortic endothelial cells (BAECs) and mice, mice, indicating that Pin1 negatively regulates vascular reactivity [23], Paneni et al. suggested that Pin1 mediates the inhibitory Ser116 phosphorylation of eNOS and reduces NO availability in human aortic endothelial cells (HAECs) [24,25] and Costantino et al. also showed that Pin1 impairs NO activity in HAECs and diabetic mouse model [26].

Hypothetically, suppression of eNOS activity and NO production by Pin 1 may attenuate the dynamic contraction-dilatation movement of cerebral blood vessels and thus result in precipitation/ deposition of plasma components onto cerebrovascular walls, thrombosis, atherosclerosis, and so on. These findings put forward a novel but detrimental Pin1-based etiological hypothesis for cerebral neuropathology, which is different from the previouslyknown beneficial roles of Pin1 in neurological disorders such as AD via suppressing amyloid- $\beta$ and tau aggregation.

\section{Future Orientations and Limitations}

In summary, this work succinctly suggests that Pin1 may aggravate thrombotic neuropathies, and Pin1 genetic polymorphism may represent a new genetic and molecular basis for neurological pathologies resulting from cerebrovascular thrombosis. For example, the SNPs rs2233678 (-842G) and rs2287839 (-5185C) might enhance Pin 1 transcription and induce atherosclerosis and thrombosis in brain vessels. Nevertheless, two major limitations of the current work should be mentioned: first, this work only provides a novel hypothesized role of Pin1 in neural vascular pathogenesis, but no sufficient experimental and statistical data were provided partially because of the current financial shortage; second, a largescale case-control study will be substantially needed to compare the putatively different distribution of Pin1 SNPs between infarct patients and age-matched non-infarct individuals, which will be essential for verifying the potential involvement of Pin1 in cerebral vascular disorders and other relevant neurological diseases.
In addition, this work may lay a new genetic basis for future precision medicine. Pin 1 gene sequencing may promote precision treatments of cerebrovascular dysfunction and ischemic cerebral diseases, including early diagnosis of high-risk individuals, precision drug development (Pin1 inhibitors), prognostic prediction, with the aim of improving current treatments for the above-mentioned neurological diseases.

\section{Acknowledgment}

This work is supported by the funding from Hebei Provincial Department of Science and Technology of China (No. 182777107D) and the Hebei Provincial Social Science Fund Projects (No. HB19SH020; No. 2019030401038).

\section{References}

1. Balgoon MJ, Ahmed GAR, Qusti SY, Shaker S (2019) Transit phases of $\beta$-amyloid and tau proteins formation and re-solubilisation in ad rat hippocampus tissue as probed by atr-ir spectroscopy. Neurology, Psychiatry and Brain Research 31: 1-8.

2. Danduga RCSR, Dondapati SR, Kola PK, Kavati SSH, Singapalli MS (2019) Combination effect of aspirin and n-acetylcysteine against global cerebral ischemic reperfusion injury in rats. Neurology Psychiatry and Brain Research 33: 101-111.

3. Shabani S, Farbood Y, Sarkaki A, Mard SA, Ahangarpour A, et al. (2019) The effect of triiodothyronine on the hippocampal long-term potentiation in an animal model of the alzheimer's disease: The role of bdnf and reelin. Neurology, Psychiatry and Brain Research 33: 82-88.

4. Sosin D, Ivashchenko D, Sozaeva Z, Ryzhikova K, Fadeeva V, et al. (2019) Cognitive impairment in patients with treatment resistant schizophrenia: Associations with drd2, drd3, htr2a, bdnf and cyp2d6 genetic polymorphisms. Neurology Psychiatry and Brain Research 33: 48-55.

5. Cao YL, Xu HC, Zhu YB, Shi MJ, Wei LX, et al. (2019) Adamts13 maintains cerebrovascular integrity to ameliorate alzheimer-like pathology. Plos Biol 17(6).

6. Ihara M (2019) Interaction between cerebrovascular disease and alzheimer pathology. Brain Pathol 29(2): 168-173.

7. Katsumata Y, Fardo DW, Kukull WA, Nelson PT (2018) Dichotomous scoring of tdp-43 proteinopathy from specific brain regions in 27 academic research centers: Associations with alzheimer's disease and cerebrovascular disease pathologies. Acta Neuropathol. Commun 6(1): 142 .

8. Sole M, Esteban Lopez M, Taltavull B, Fabregas C, Fado R, et al. (2019) Blood-brain barrier dysfunction underlying alzheimer's disease is induced by an ssao/vap-1-dependent cerebrovascular activation with enhanced a beta deposition. Bba-Mol Basis Dis 1865(9): 2189-2202.

9. Raghib MF, Mutzenbach JS, Rosler C, Otto F, Mc Coy M, et al. (2018) Acute treatment of stroke due to spontaneous calcified cerebral emboli causing large vessel occlusion. J Clin Neurosci 47: 56-61.

10. Diacinti D, Cartocci G, Colonnese C (2018) Cerebral venous thrombosis: A case series and a neuroimaging review of the literature. J Clin Neurosci 58: 142-147.

11. Akpinar S, Gelener P (2018) Endovascular treatment of acute tandem occlusion strokes and stenting first experience. J Clin Neurosci 47: 328331.

12. Rosen C, McKetton L, Russell J, Sam K, Poublanc J, et al. (2018) Longterm changes in cerebrovascular reactivity following ec-ic bypass for intracranial steno-occlusive disease. J Clin Neurosci 54: 77-82.

13. Moscote Salazar L (2018) Role of early partial recanalization and intravenous thrombolysis for acute ischemic stroke. J Clin Neurosci 52: 168. 
14. Wang JZ, Zhang YH, Bai J, Liu YW, Du WT (2019) Pin1, a perspective on genetic biomarker for nonalcoholic fatty liver disease (nafld). Metabolism Open 3: 100014.

15. Ma SL, Tang NL, Tam CW, Lui VW, Lam LC, et al. (2012) A pin1 polymorphism that prevents its suppression by ap4 associates with delayed onset of alzheimer's disease. Neurobiol Aging 33(4): 804-813.

16. Lu J, Yang L, Zhao H, Liu B, Li Y, et al. (2011) The polymorphism and haplotypes of pin1 gene are associated with the risk of lung cancer in southern and eastern chinese populations. Hum Mutat 32(11): 12991308.

17. Zeng LY, Luo SQ, Li X, Lu MX, Li HH, et al. (2017) Functional pin1 promoter polymorphisms associated with risk of nasopharyngeal carcinoma in southern chinese populations. Sci Rep-UK 7: 4593.

18. Wang JZ, Liu GJ, Li ZY, Wang XH (2016) Pin1 in cardiovascula dysfunction: A potential double-edge role. Int J Cardiol 212: 280-283.

19. Hou H, Wang JZ, Li XM (2015) The role of the peptidyl-prolyl cis/trans isomerase pin 1 in the occurrence and development of alzheimer's disease. Prog Biochem Biophys 42(5): 434-442.

20. Wang JZ, Zhang Y (2015) Configuration-specific immunotherapy targeting cis pthr231-pro232 tau for alzheimer disease. J Neurol Sci 348(1-2): 253-255.

\section{ISSN: 2574-1241}

DOI: 10.26717/BJSTR.2019.22.003756

Jing-Zhang Wang, Jing Bai, Yu-Hua Zhang. Biomed J Sci \& Tech

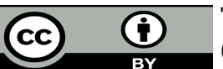

This work is licensed under Creative

Commons Attribution 4.0 License

Submission Link: https://biomedres.us/submit-manuscript.php
21. Lonati E, Brambilla A, Milani C, Masserini M, Palestini P, Bulbarelli A (2014) Pin1, a new player in the fate of hif-1 alpha degradation: An hypothetical mechanism inside vascular damage as alzheimer's disease risk factor. Front Cell Neurosci 8: 001.

22. Wang JZ (2018) A novel glucose-pin1-enos-no signaling axis links diabetes mellitus with cardiovascular diseases. Int J Cardiol 271: 262.

23. Ruan L, Torres CM, Qian J, Chen F, Mintz JD, et al. (2011) Pin1 prolyl isomerase regulates endothelial nitric oxide synthase. Arterioscler Thromb Vasc Biol 31(2): 392-398.

24. Paneni F, Costantino S, Battista R, Capretti G, Castello L, et al. (2013) Prolyl-isomerase-1 (pin1) mediates hyperglycemia-induced oxidative stress, endothelial function and vascular inflammation: Insights in patients with type 2 diabetes. Eur Heart J 34: 604-604.

25. Paneni F, Costantino S, Castello L, Battista R, Capretti G, et al. (2015) Targeting prolyl-isomerase pin 1 prevents mitochondrial oxidative stress and vascular dysfunction: Insights in patients with diabetes. Eur Heart J 36(13): 817-828.

26. Costantino S, Paneni F, Luscher TF, Cosentino F (2016) Pin1 inhibitor juglone prevents diabetic vascular dysfunction. Int J Cardiol 203: 702707.

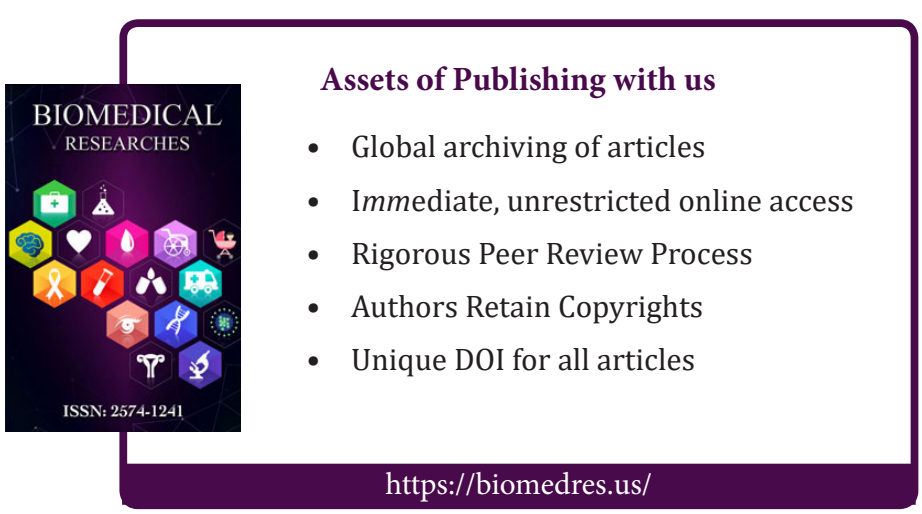

